\title{
FORUM
}

\section{Neue Dynamik für konkrete Ergebnisse Barcelona-Prozess: Union für den Mittelmeerraum}

\author{
Günter Gloser*
}

In diesen Wochen wird uns wieder vor Augen geführt, welche Folgen Unfrieden und Instabilität in unserer unmittelbaren Nachbarschaft haben können. Ganz Europa, ob im Süden oder im Osten, ist von den Entwicklungen im Kaukasus unmittelbar betroffen. Uns wird vor Augen geführt, wie wichtig es ist, dass die Europäische Union geschlossen und einheitlich auftritt, und dass auch alle Mitgliedstaaten dieses Auftreten mittragen, auch und gerade in der Frage der Definition der Beziehungen zu unseren Nachbarländern. Für die regionale Zusammenarbeit mit unseren Mittelmeerpartnern haben wir hierfür bereits Mitte der 1990er Jahre gemeinsam mit unseren europäischen Partnern - insbesondere Frankreich und Spanien - den Barcelona-Prozess als Instrument euro-mediterraner Zusammenarbeit ins Leben gerufen. Zugegeben, der Barcelona-Prozess hatte in seiner praktischen Umsetzung offenkundig auch Schwächen. Aber die Notwendigkeit der Zusammenarbeit mit unseren Mittelmeerpartnern stand immer außer Frage.

Unsere Bemühungen um eine substanzielle Stärkung der Zusammenarbeit waren daher nur konsequent. So hat der französische Staatspräsident Sarkozy im Vorfeld der französischen EU-Ratspräsidentschaft die Initiative ergriffen. Deutsches Verständnis und Bestreben war es dabei, diese Fortentwicklung des Barcelona-Prozesses als gesamteuropäische Aufgabe fortzuführen.

Der gemeinsame deutsch-französische Ansatz wurde vom Europäischen Rat im März 2008 indossiert, von der Europäischen Kommission konkretisiert und war maßgeblich für den Erfolg des Mittelmeergipfels am 13. Juli 2008 in Paris. Der in der Gipfelerklärung zum Ausdruck gekommene Konsens aller teilnehmenden Länder, in dieser Form den BarcelonaProzess weiterzuentwickeln, ist für alle Beteiligten ein großer Erfolg und legt den Grundstein für die beim kommenden Außenminister-Treffen im November 2008 noch zu klärenden Fragen.

Klar ist jedoch bereits jetzt: Kennzeichnend für die Zusammenarbeit im Mittelmeerraum ist die gemeinsame Verantwortung aller in einer gleichberechtigten Partnerschaft. Diese Verantwortung drückt sich aus in einer stärkeren politischen Wahrnehmung und durch konkrete, sichtbare und substanzielle Projekte.

\section{Das Mittelmeer geht uns alle an}

Die Auswirkungen der Kaukasus-Krise auf die globale Sicherheitsarchitektur sind inzwischen evident. Auch Konflikte in der Mittelmeerregion beeinträchtigen die Sicherheit des gesamten euro-mediterranen Raums und darüber hinaus. Während es im Osten vornehmlich noch immer um die Bewältigung der Folgen des Kalten Krieges geht, spielen im Süden asymmetrische Herausforderungen, die häufig mit religiösen Argumenten gerechtfertigt

\footnotetext{
* Günter Gloser, MdB, Staatsminister für Europa, Auswärtiges Amt.
} 
werden, eine herausgehobene Rolle. Frieden und Stabilität in der Nachbarschaft liegen im ureigensten Interesse Europas.

Mit Entsetzen müssen wir jeden Sommer wahrnehmen, wie zahlreiche Menschen im Mittelmeer auf der Suche nach einem besseren Leben in Europa - in Frankreich, Deutschland oder sonst einem Land, in dem sie sich bessere Chancen erhoffen - zu Tode kommen. Im Rahmen der Zusammenarbeit im Mittelmeerraum spielt daher das Thema Migration eine zentrale Rolle. Eine zielorientierte Migrationspolitik, die Verbesserung der Rahmenbedingungen in den Herkunftsländern sowie die Bekämpfung illegaler Migration bei gleichzeitiger Betonung des Flüchtlingsschutzes können nur auf Basis verstärkter partnerschaftlicher Zusammenarbeit erreicht werden; eine solche Strategie verfolgt die Europäische Union mit dem ,Gesamtansatz Migration“. Hierzu hatte man sich bereits bei der ersten dem Thema ,Migration' gewidmeten euro-mediterranen Ministerkonferenz im November 2007 in Portugal auf eine Erklärung geeinigt, die hoffnungsvoll stimmte, nun aber tatsächlich umgesetzt werden muss.

Im Bereich der Energie haben Lieferschwierigkeiten im Osten ebenso unmittelbare Auswirkungen auf die gesamte Energieversorgung der Europäischen Union wie - positiv gewendet - die zusätzliche Erschließung von Energiequellen im Süden. Diversifizierung und langfristige Sicherung der Energieversorgung ist das gemeinsame Interesse aller EU-Länder. Der gesamte Mittelmeerraum wird auch in diesem Zusammenhang eine immer wichtigere Rolle einnehmen.

Diversifizierung kann uns auch bei unserem Ziel der Senkung der Treibhausgasemissionen helfen. Die Europäische Union hat unter deutscher EU-Ratspräsidentschaft im Frühjahr 2007 feste, verbindliche Reduktionsziele beschlossen. Hier müssen wir auch bei unseren Partnern auf entsprechende Anstrengungen hinwirken. Der nicht mehr aufzuhaltende Klimawandel wird darüber hinaus die ohnehin schon vorhandene Trinkwasserknappheit in den Mittelmeerländern weiter verschärfen.

All diese Beispiele verdeutlichen auch die gesamteuropäische Verantwortung. Wir können es uns heute nicht mehr leisten, unsere Interessen gegenüber anderen Weltregionen ausschließlich national zu definieren. Ohne eine einheitliche europäische Außenpolitik werden wir nicht in der Lage sein, unsere Interessen hinreichend wahrzunehmen. Auch hierzu brauchen wir den Vertrag von Lissabon.

Gemeinsame Verantwortung bedeutet aber auch, dass sich alle - auch die Mittelmeerpartner - bemühen müssen, die vorhandenen Spannungen untereinander abzubauen. Die Union für das Mittelmeer soll - aufbauend auf dem Barcelona-Acquis - eine pragmatische Zusammenarbeit ermöglichen, die zu spürbaren Verbesserungen für die Menschen im Mittelmeerraum führt.

\section{Gleichberechtigte Partnerschaft}

Ein weiteres Kernelement der Zusammenarbeit ist die gleichberechtigte Partnerschaft. Wir wollen eine gleichberechtigte Zusammenarbeit mit allen Mittelmeerpartnern - im Maghreb und Maschrek, mit Israel und - nicht zu vergessen - mit den nördlichen Mittelmeeranrainern, die nicht Mitglied der Europäischen Union sind. Die Bezeichnung „Union für den Mittelmeerraum“ darf dabei jedoch nicht darüber hinwegtäuschen, dass sie - im Gegensatz zur Europäischen Union - nicht auf Integration, sondern auf Kooperation aufgebaut ist. Eine Kooperation, die allerdings zwingend auf gleicher Augenhöhe gestaltet werden muss. An der partnerschaftlichen Zusammenarbeit der Europäischen Union mit allen Mittelmeerpartnern ändert sich auch nichts dadurch, dass die deutsch-französische Lokomotive diesen Zug auf das richtige Gleis gesetzt hat. 
Beim Gipfel am 13. Juli 2008 in Paris wurde die Schaffung eines gemeinsamen Ko-Vorsitzes bestätigt - jeweils ein Vertreter der Europäischen Union und ein Vertreter der Partnerländer. So übernahmen zunächst Frankreich und Ägypten den Vorsitz. Während für den Vorsitz der Partnerländer der Vorsitz für zwei Jahre gewählt wird, muss der Vertreter der Europäischen Union im Einklang mit den geltenden Verträgen bestimmt werden. Die deutsch-französische Einigung sieht vor, dass zunächst auch hier ein zweijähriger Rhythmus gelten soll. Der gemeinsame Ko-Vorsitz ist ein Symbol für die neue Solidarität zwischen den Staaten südlich und nördlich des Mittelmeers, die Südanrainer werden aus der reinen ,Nehmer-Rolle' oder - so eine andere Sicht - aus der Bevormundung heraus in die gemeinsame Verantwortung eingebunden.

Unterstützt werden soll der gemeinsame Vorsitz von einem Sekretariat. Auch hier streben wir eine paritätische Zusammensetzung an. Über die konkrete Ausgestaltung des Sekretariats sollen im November 2008 die Außenminister entscheiden. Neben der noch offenen Frage des Sitzes geht es dabei vor allem um die Ausgestaltung des Mandats. Während bei der Sitzfrage gerade die gleichberechtigte Partnerschaft zum Tragen kommen muss, werden wir bei der Ausgestaltung des Mandats darauf zu achten haben, dass wir das Sekretariat nicht politisch überfrachten. Um konkrete Ergebnisse zu erzielen, muss das Sekretariat schlank und projektorientiert arbeiten. Durch Einrichtung des Sekretariats sollte weder zusätzliche Bürokratie verursacht noch lähmende politische Konflikte in die Projektarbeit hineingetragen werden. Dies würde schon die bloße Projektidentifizierung wesentlich erschweren.

Mit einer solchen Ausgestaltung des Sekretariats ist nicht gemeint, dass wir den Barcelona-Acquis vernachlässigen. Die gemeinsamen Werte, auf die wir uns zu Beginn des Barcelona-Prozesses verständigt haben, bleiben weiterhin zentraler Maßstab. Dazu gehört vordringlich, den Rechtsstaat und die Achtung der Menschenrechte zu fördern. ,Auf gleicher Augenhöhe‘ mit unseren Mittelmeerpartnern zusammenzuarbeiten, kann nicht bedeuten, diese Werte zu negieren. Das politische Mandat muss jedoch auf politischer Ebene bleiben. Mit dem „Gemischten Ständigen Ausschuss“ werden wir zudem über ein Gremium verfügen, das kontinuierliche politische Arbeit gewährleistet.

\section{Stärkere politische Wahrnehmung}

Der Barcelona-Prozess hat in den vergangenen Jahren oft darunter gelitten, dass er politisch nicht ausreichend stark in der öffentlichen Debatte sichtbar war. Hier haben wir - dank der intensiven Anstrengungen Frankreichs - in den letzten Monaten schon viel erreicht. Die Ausrichtung des Mittelmeergipfels zu Beginn der französischen EU-Ratspräsidentschaft war ein großer Erfolg - für Europa und für alle Mittelmeerpartner. Zukünftig sollen die Staatsund Regierungschefs regelmäßig - alle zwei Jahre - zu einem Gipfeltreffen zusammenkommen. Der nächste Gipfel ist somit zum 15-jährigen Bestehen des Barcelona-Prozesses vorgesehen.

\section{Konkrete und substanzielle Projekte}

Kernpunkt der inhaltlichen Fortentwicklung des Barcelona-Prozesses ist die Umsetzung konkreter und substanzieller Projekte. Die Staats- und Regierungschefs haben sich bei dem Gipfel im Juli 2008 bereits auf sechs Projektbereiche geeinigt. Entscheidend ist nun, die vorhandene Dynamik zu nutzen und konkrete Projekte tatsächlich umzusetzen - Projekte, die den Menschen die Vorteile der Zusammenarbeit im Mittelmeerraum unmittelbar nahebringen. 
Deutschland engagiert sich stark im Bereich des Ausbaus erneuerbarer Energien und hat für den Bereich der Solarenergie bereits zu Beginn der Verhandlungen einen entsprechenden Vorschlag eingebracht. Dabei geht es um nicht weniger als den umfassenden Ausbau aller Solartechniken mit Schwerpunkt auf solarthermischen Kraftwerken in den südlichen Mittelmeeranrainerstaaten sowie - zu gegebener Zeit - der Errichtung eines inter-mediterranen Stromnetzwerks. Ziel ist die Senkung der Treibhausgasemissionen, die Minderung der Energieabhängigkeit und -armut der Länder, die Sicherung der europäischen Energieversorgung und die Förderung der wirtschaftlichen und industriellen Entwicklung. Für die deutsche Wirtschaft bieten sich hier gute Chancen für eine aktive Beteiligung. Gerade auf dem Gebiet der Umwelttechnologien sind deutsche Unternehmen führend.

Weitere Projektbereiche sind unter anderem die Säuberung des Mittelmeers und der Ausbau der Verkehrsinfrastruktur (Meer- und Landverkehrswege) im und um das Mittelmeer. Andererseits müssen wir aber auch darauf achten, dass solche Projekte, die innerhalb der Europäischen Union in der Vergangenheit keine Mehrheit gefunden haben, nicht über die Hintertür erneut eingebracht werden.

Entscheidend für eine erfolgreiche Verwirklichung der vorgesehenen Projekte ist der Grundsatz variabler Geometrie. Während institutionelle Fragen wie Ko-Vorsitz oder Sitz des Sekretariats im Konsens beschlossen werden müssen, liegt der Charme der Projektarbeit mit variabler Geometrie darin, dass sich interessierte Länder zusammentun und Projekte pragmatisch, konstruktiv und frei von lähmenden politischen Hindernissen tatsächlich verwirklichen.

Erste Schritte sind getan, der Zusammenarbeit in guter Atmosphäre neuen Schwung zu verleihen. Wichtig ist es, das Momentum zu bewahren und in dem kooperativen Geist, der die Gipfelerklärung auszeichnet, weiter an der Lösung der konkreten institutionellen Fragen zu arbeiten.

Nun steht die Klärung noch offener institutioneller Fragen an, die auf dem Außenministertreffen am 3. und 4. November 2008 in Marseille entschieden werden sollen. Gemeinsames Ziel ist es, vor Ende 2008 uneingeschränkt arbeitsfähige Strukturen zu schaffen, damit möglichst rasch konkrete und sichtbare Projekte umgesetzt werden können.

Die stark projektorientierte und damit pragmatisch ausgerichtete Fortentwicklung des früheren Barcelona-Prozesses kann ein wesentliches Aktionsfeld deutscher Politik in den nächsten Jahren werden. Gerade weil er sich auf genau umrissene und größtenteils unstreitige Vorhaben konzentriert, kann der „Barcelona-Prozess: Union für den Mittelmeerraum“ neuen Schwung in die Partnerschaft der Europäischen Union mit den Mittelmeerpartnern bringen. Mit einer nachhaltigen wirtschaftlichen Entwicklung trägt er zu politischen Fortschritten und zur friedlichen Beilegung von Konflikten in der Region bei.

Der Rahmen für eine Zusammenarbeit ist geschaffen, der Erfolg hängt nun vom Willen aller Partner ab. Deutschland wird mit aller Kraft für ein Gelingen arbeiten. 